\title{
A SUBORDINATED STOCHASTIC PROCESS MODEL \\ WITH FINITE VARIANCE FOR SPECULATIVE PRICES
}

\author{
by \\ Peter King Clark \\ Discussion Paper No. 1, April 1971
}

\author{
Center for Economic Research \\ Department of Economics \\ University of Minnesota \\ Minneapolis, Minnesota 55455
}




\section{Acknowledgements}

Thanks are due to Hendrik Houthakker and Christopher Sims, for both encouragement and advice in developing this paper. As usual, all remaining errors are my own. This research was supported by a Harvard Dissertation Fellowship, NSF grant 33-708, and the Boston College Department of Economics. 


\section{A SUBORDINATED STOCHASTIC PROCESS MODEL}

\section{WITH FINITE VARIANCE FOR SPECULATIVE PRICES}

\section{INTRODUCTION}

The past seventeen years have seen a large amount of research by academic economists on prices in speculative markets, an area which was formerly studied almost exclusively by financial speculators and. their advisors. ${ }^{1}$ Considering the time series of prices at short intervals on a speculative market such as that for futures in commodities, or corporation shares, one primary characteristic is evident. If $X_{t}$ denotes price at time $t$ and $\Delta X_{t}=X_{t}-X_{t-1}$, examination of the data suggests that:

$$
E\left(\Delta X_{t}\right)=0 \text { and } E\left(\Delta X_{t} \cdot \Delta X_{t}\right)=0 t \neq s
$$

The increments in the price process are stationary in the mean and uncorrelated; a random walk model (1)

$$
\text { (1) } \mathrm{X}_{\mathrm{t}}=\mathrm{X}_{\mathrm{t}-1}+\varepsilon_{\mathrm{t}}, E\left(\varepsilon_{\mathrm{t}}\right)=0, E\left(\varepsilon_{\mathrm{t}} \varepsilon_{\mathrm{t}}\right)=0, t \neq \mathrm{s}
$$

explains these empirical facts well.

Besides empirical realism, the random walk model has a theoretical basis. $^{3}$ If price changes were correlated, then alert speculators should notice the correlation and trade in the right direction until the relationship was removed. This was first shown by Bachelier in 1900, when he derived the diffusion equation from a condition that speculators should receive no information from past prices. Equation (1) is, of course, a solution to a discrete formulation of the diffusion problem.

1. See Clark [6] for a comprehensive bibliography, or Cootner [8] for a collection of these articles.

2. Bachelier [3] 
It is also empirically evident that the price changes $\Delta \mathrm{X}_{\mathrm{t}}$, however independent, are not normally distributed. Instead of having the normal shape, which would be the case if the components in $\Delta \mathrm{x}_{t}$ were almost independent and almost identically distributed, ${ }^{3} \Delta X$ has too many small and too many large observations, as pictured in Figure 1.

One way to express this is to say that the distribution of $\Delta \mathrm{X}$ is leptokurtic, for the sample kurtosis:

$$
\hat{\mathrm{k}}_{\Delta \mathrm{X}}=\frac{\frac{1}{\mathrm{n}} \sum_{1}\left(\Delta \mathrm{X}_{1}-\overline{\Delta \mathrm{X}}\right)^{4}}{\frac{1}{\mathrm{n}} \sum_{1}\left[\left(\Delta \mathrm{X}_{1}-\overline{\Delta \mathrm{X}}\right)^{2}\right]^{2}}
$$

is much greater than 3, the value for a normal population.

It is evident, then, that conditions sufficient for the Central Limit Theorem are not met by the influences which make up $\Delta x$. The violation of these conditions and the reason for the leptokurtic distribution of $\Delta \mathrm{X}$ is the subject of the present article.

In 1963, Mandelbrot set out to explain this non-normality in price changes that had been observed by Kenda $11^{4}$ and many others. 5 The observed distribution of price changes clearly indicates that the Central Limit Theorem does not apply to them. But what condition is being violated? Mandelbrot decided that the individual effects making up a price change did not have finite variance, but were still independent. The distribution of price change should then belong to the stable family

3. Feller [9], Gnedenko and Kolmogorov [10], and Loève [13] contain good expositions on the conditions under which the Central Limit Theorem is satisfied.

\section{Kenda11 [11]}

5. Mandelbrot [14] lists many references to the problem of nonnormality, one as early as 1915 . 


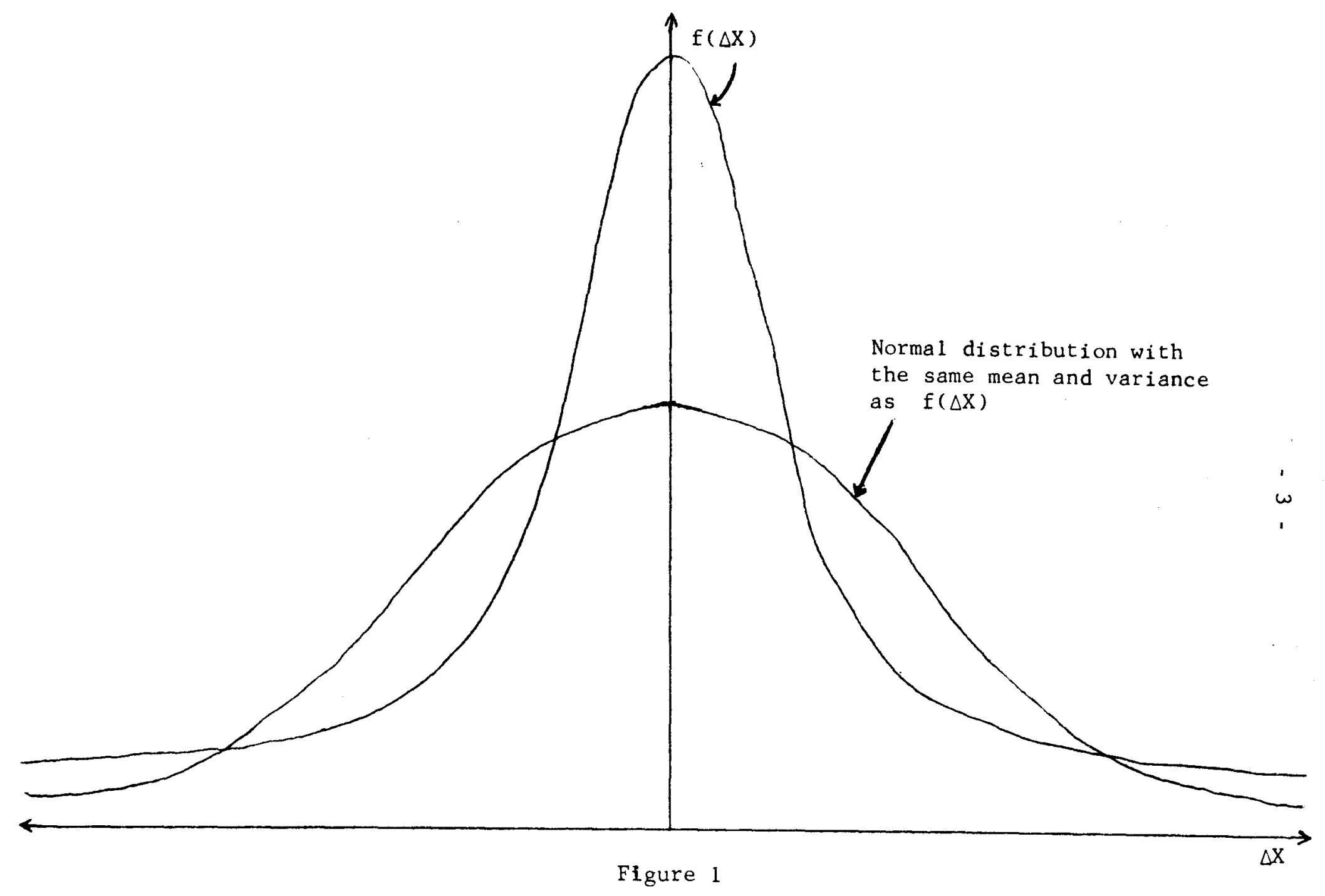

Distribution of $\Delta \mathrm{X}$, the daily changes in price 
of distributions, which were shown by Levy to be the only possible limit distributions for sums of independent random variables. ${ }^{6}$ These stable distributions have an unbounded kurtosis and will usually give high values for any measured sample kurtosis, thus making them good candidates for the distribution of price change.

The opposing hypothesis presented and tested in this paper is that the distribution of price change is subordinate ${ }^{7}$ to a normal distribution. The price series for cotton futures evolves at different rates during identical intervals of time. The number of individual effects added together to give the price change during a day is variable and in fact random, making the standard Central Limit Theorems inapplicable.

The different evolution of price series on different days is due to the fact that information is available to traders at a varying rate. On days when no new information is available, trading is slow, and the price process evolves slowly. On days when new information violates old expectations, trading is brisk, and the price process evolves much faster.

\section{DISTRIBUTIONS SUBORDINATE TO THE NORMAL DIETRIBUTION}

\section{A. The Central Limit Theorem.}

As we have noted in the last section, empirical evidence shows that the random elements that make up cotton futures price differences do not obey conditions sufficient for the Central Limit Theorem to apply.

6. Levy. [12]. Gnedenko and Kolmogorov [10] is the classic exposition in this field.

7. For a definition and explanation of subordination, see Section II. 
In the following development, the Central Limit Theorem is generalized in a way that makes the resulting limit distributions applicable to the distribution of cotton futures price differences.

First, one variant of the Central Limit Theorem and two Lemmas are stated without proof $^{8}$

$\underline{\text { Lemma } 1}$ For $n=1,2, \ldots$, and $t>0$,

$$
\left|e^{1 t}-1-i t-\frac{(i t)^{2}}{2 !}-\cdots-\frac{(1 t)^{n-1}}{(n-1) !}\right| \leq \frac{t^{n}}{n !}
$$

That is, the Taylor Series expansion for $e^{l^{t}}$ differs from $e^{t^{t}}$ by less than the first excluded term.

Lemma 2 Let $m_{n}=\int_{-\infty}^{\infty} y^{n} d F_{y}$, and $M_{n}=\int_{-\infty}^{\infty}|y|^{n} d F_{y}$, where $y$ is a random variable and $F_{y}$ is its distribution; $m_{z}$ and $M_{z}$ are extended real numbers. If $M_{n}<\infty$, then $n^{t h}$ derivative of $\Phi=E\left(e^{1 \times y}\right)$, the characteristic function of $y$, exists and is a continuous function given by: $\underbrace{(\infty)}(w)=1^{n} \int_{-\infty}^{\infty} e^{1 w y} y^{n} d F_{y}$.

Corollary If $\mathrm{m}_{z}<\infty$ then ${ }^{\prime}(0)=\mathrm{im}$, and $\mathbf{\prime}^{\prime \prime}(0)=-\mathrm{m}_{R}$.

Theorem 1 (Central Limit Theorem) Let $\left\{Y_{1}\right\}$ be a sequence of identically distributed independent random variables with mean 0 and variance 1 . Let $S=\sum_{1=1}^{n} Y_{1}$. Then the distri bution of $S_{n} \sqrt{n}$ tends to the unit normal distribution.

First, we may generalize this theorem to the case where the number of terms, $n$, in the sum $s_{n}$ is itself a random variable.

8. See Feller [9] for proofs of both Lemmas and a slightly modified form of Theorem 1 . 
Theorem $2^{9}$ Let $\left\{\mathrm{N}_{\mathrm{n}}\right\}$ be a sequence of positive integral valued random variables obeying the property $\operatorname{plim}_{n \rightarrow \infty}\left(\frac{N_{n}}{n}\right)=1$. Let $\left\{Y_{1}\right\}$ have the same distribution as in Theorem 1 , and $S_{N_{n}}=\sum_{1=1}^{N_{n}} Y_{1}$. Assume $\left\{N_{1}\right\}$ and $\left\{Y_{1}\right\}$ are mutually independent. Then $S_{N_{n}} / \sqrt{n}$ converges in probability to the unit normal distribution as $n \rightarrow \infty$.

Proof: It must be shown that $\Phi^{N_{n}}(w / \sqrt{n})$, the characteristic function of $s_{N_{a}} / \sqrt{n}$, approaches $e^{-n^{2} / 2}$, the characteristic function of a unit normal variable, as $n \rightarrow \infty$.

$$
\Phi(w / \sqrt{n})=\int_{-\infty}^{\infty} e^{i(w / \sqrt{n}) y} d F_{y}
$$

By Lemma 1 and the Corollary to Lemma 2, $\Phi(w / \sqrt{n})=1-\frac{w^{2}}{2 n}+o\left(\frac{1}{n}\right)$ as $n \rightarrow \infty . \quad \Phi^{N a}(w / \sqrt{n}) \rightarrow-\frac{N_{n}}{2 n} w^{2}$ as $n \rightarrow \infty$, and $\log \Phi^{N_{n}}(w / \sqrt{n}) \rightarrow\left(\frac{N_{n}}{n}\right)$. $\left(-\frac{w^{2}}{2}\right), n \rightarrow \infty$. Since by hypothesis $\left(\frac{N_{n}}{n}\right) \underset{\text { prob }}{ } 1, n \rightarrow \infty, \Phi^{N_{n}}(w / \sqrt{n}) \overrightarrow{\text { prob }}$ $\mathrm{e}^{-\mathrm{w}^{2} a}, \mathrm{n} \rightarrow \infty$, and the theorem is proved.

This theorem says that if $N_{a}$ has small variation around $n$ for large $\mathrm{N}_{\mathrm{n}}$ $n$, then $\sum_{1=1} Y_{1}$, the random sum of random variables, still approaches the normal distribution.

Now suppose that $N_{n}$ has appreciable variance around $n$ even for large n. This is the case that is relevant for the cotton futures price process. The number of small price changes added up on each day is variable. For instance, let: $N_{n}=[\mathrm{Zn}]$ where $Z$ has mean 1 and variance $\Gamma>0$, where [ ] denotes "largest integer less than". Following the development in Theorem 2, for large $n$,

$$
\begin{aligned}
N_{n} \log \Phi(w / \sqrt{n}) & \sim \frac{[Z n]}{n}\left(-\frac{1}{2} w^{2}\right), n \rightarrow \infty \\
\Phi^{N_{n}}(w / \sqrt{n}) & \sim e^{-Z w^{2}} / 2, n \rightarrow \infty
\end{aligned}
$$

9. The proof of a theorem similar to this was given by Robbins [18]. See also Anscombe [2], Billingsley [4], Renyi [16], [17], and Feller [9] for theorems on limit distributions of random sums of random variables. 
This characteristic function may be inverted to find the limit distribution of $S_{N_{n}} \sqrt{n}$. This is the characteristic function for a variable with random variance $z$; as we shall see, the distribution for such a variable is not normal, and depends on the distribution of $z$.

We have just proved the following theorem:

Theorem 3 Let $\left\{\mathrm{Y}_{1}\right\}$ be distributed as in Theorem 1 , and let

$$
\mathrm{S}_{\mathrm{N}_{\mathrm{n}}}=\sum_{1=1}^{\mathrm{N}_{\mathrm{n}}} \mathrm{X}_{1}
$$

Let $N_{n}=[\mathrm{Zn}]$ for large $n$, where $Z$ is a random variable with mean 1 , again independent of $\left\{\mathrm{Y}_{1}\right\}$. Then $\mathrm{S}_{\mathrm{N}_{\mathrm{n}}} / \sqrt{n}$ has $f(u)=\frac{1}{\sqrt{2 \pi Z}} e^{-u^{2} / 2 Z}$ as its density.

We now have the limit distribution of $S_{N_{n}}$ conditional on $Z$. Over a long period of time, the price changes of cotton futures will be the marginal distribution of $S_{N_{n}}$ found by taking the expectation of the distribution above with respect to $Z$. This simple procedure yields the subordinate distributions described below.

\section{B. Subordinated Stochastic Processes}

Discrete stochastic processes are indexed by a discrete variable, usually time, in a straightforward manner: $x_{0}, x_{1}, \ldots, x_{t}, x_{t+1}, \ldots$. This may also be written $X(0), X(1), \ldots X(t), \ldots ; X(s)$ is the value that a particular realization of the stochastic process assumes at time $s$. Instead of Indexing by the integers $0,1,2, \ldots$, the process could be indexed by a set of numbers $t_{1}, t_{2}, t_{3}, \ldots$, where these numbers were themselves a realization of a stochastic process (with positive increments, so that $\left.t_{1} \leq t_{2} \leq t_{3} \leq \ldots\right)^{11}$. That is, if $T(t)$ is a

10. See Robbins [18] for a different result on the limit distribution of a random sum of random variables.

11. The idea of a subordinated process was originated by Bochner [5] However, for a simpler exposition, see Feller [9], p. 333 ff. 
positive stochastic process, a new process $X(T(t))$ may be formed. This process is said to be subordinated to $X(t) ; T(t)$ is called the directing process. The distribution of $\Delta X(T(t))$ is said to be subordinate to the distribution of $\Delta \mathrm{X}(t)$.

Note that $\Delta X(t)$ will assume the role of the individual effects In the evolution of the price process, while $T(t)$ is a clock measuring the speed of evolution. $X(T(t))$ is, of course, the price process itself. The following theorem holds for very general classes of subordinated stochastic processes with independent increments. Aside from providing a simple formula for calculating the variance of the increments, it also shows that this variance is finite for processes having increments with finite variance, and directed by a process with increments of finite mean.

Theorem $4^{12}$ Let $X(t)$ and $T(t)$ be processes with stationary independent increments; that is:

1. $X\left(t_{k+1}\right)-X\left(t_{k}\right),(k=1,2, \ldots, n-1)$, are mutual1y independent for any finite set $t_{1}<\ldots \ldots \leq t_{2}$, and similarly for $T(t)$.

2. $X(s+t)-X(s)$ depends on $t$ but not on $s$ for all $s$, and similarly for $T(t)$.

Let the increments of $X(t)$ be drawn from a distribution with mean 0 and finite variance $\sigma^{2}$; i.e., $E[X(s+1)-X(s)]=0$, all $s$, and $E[X(s+1)$ $X(s)]^{2}=\sigma^{2}$, all s. Let the increments of $T(t)$ be drawn from a positive distribution with mean $\alpha$, independent of the increments of $X(t)$. That is, $E[T(s+1)-T(s)]=\alpha$, and $[T(s+t)-T(s)] \geq 0, t>0$. Then the subordinated stochastic process $X(T(t))$ has stationary independent increments

12. Robbins [18] proves this theorem in somewhat less generality. 
with mean 0 and variance $\alpha \sigma^{2}$.

Proof: If the steps $\Delta X(t)$ are independent with mean 0 and variance $\sigma^{3}$, then $v$ steps have mean 0 and variance $v \sigma^{2}$. Therefore the variance of $\Delta X(T(t))$ conditional on $\Delta T(t)$ is

$$
\text { Var }(\Delta X(T(t)) \mid \Delta T(t)=v)=v \sigma^{2} \text {. }
$$

The unconditional variance of $\Delta X(T(t))$ is just the expectation of the conditional variance.

$$
E_{\Delta(T(t))}\left(v \sigma^{3}\right)=\alpha \sigma^{2}
$$

The expectation of the mean of the steps over the distribution of $\Delta \mathrm{T}(t)$ is clearly 0 .

Note that no mention has been made of the variance of the increments of the directing process; this says that if the directing process has a finite mean, then $\Delta X(T)$ will have a finite variance unless $\Delta T$ does not. It also indicates that even if the parameters $\sigma^{2}$ and $\alpha$ are specified, a family of distributions with 0 mean and identical variance may be obtained by allowing the variance or other parameters of distribution of $\Delta T(t)$ to change.

Corollary 4.1: If $X(t)$ is normal with stationary independent increments, and $T(t)$ has stationary independent positive increments with finite second moment which are independent of $x$, then the kurtosis, $k$, of the increments of $X(T(t))$ is an Increasing function of the variance of the increments of $T(t)$.

Proof: The kurtosis $\mu_{4} / \sigma^{4}$ for a normal distribution is 3 . Therefore conditional on $\Delta T(t)=v$

$$
E\left(\Delta X(T(t) \mid \Delta T(t)=v)=3 v^{2} \sigma^{4}\right.
$$


The unconditional expectation is

$$
E_{\Delta T(t)}\left(3 v^{2} \sigma^{4}\right)=3 \sigma^{4}\left(\alpha^{2}+\operatorname{Var}(v)\right)
$$

The unconditional kurtosis is then

$$
\text { (2) } k_{\Delta X(T(t))}=\frac{3 \sigma^{4}\left(\alpha^{2}+\operatorname{Var}(v)\right)}{\alpha^{2} \sigma^{4}}=3\left[\frac{\alpha^{2}+\operatorname{Var}(v)}{\alpha^{3}}\right]
$$

where $\alpha$ is the mean of $v$, the random variable which represents the increments of $T(t)$.

Note that this corollary shows that the introduction of any directing process makes the distribution of the increments of $X(T(t))$ only more leptokurtic. The corollary is directly applicable to the limit distributions found in Theorem 3 , since we know that the limit distribution of a random sum of random variables which obey the Central Limit Theorem is asymptotically normal with random variance, or in new terminology, subordinate to the normal distribution.

Corollary 4.2: If the condition that the increments of $T(t)$ are stationary and independent is removed, then Theorem 4 still holds, with the exception that the increments of $X(T(t))$ are uncorrelated as opposed to independent.

As has been pointed out by Mandelbrot and Taylor ${ }^{13}$, in certain cases distributions subordinate to a normal distribution have symmetric stable distributions. Feller ${ }^{14}$ shows that if $X(t)$ and $T(t)$ have stationary mutually independent increments, when $\Delta X(t)$ has a symmetric stable distribution with $1<\alpha_{1} \leq 2,15$ and $\Delta T(t)$ has a stable distribution with

13. Mandelbrot and Taylor [15].

14. Feller $[9]$.

15. $\alpha$ is a parameter of stable distributions, $0<\alpha \leq 2$. Stable distributions behave asymptotically like $f(u) \sim|u|^{-(\alpha+1)}$ for large $u$, so that for $\alpha \leq 1$ they have no mean, and for $\alpha<2$ they have no variance. 
$0<\alpha_{2}<1$, then $\Delta X(T(t))$ has a stable distribution with $\alpha=\alpha_{1} \cdot \alpha_{2}$. Note that this result fits intuitively with Theorem 4 . If $\alpha_{1}=2$, and $\Delta X(T(t))$ therefore has a distribution subordinate to a normal distribution, the variance of this distribution is finite if the mean of the distribution of $\Delta \mathrm{T}(t)$ is finite, but is infinite in this case, where $F_{T}$ has no mean.

C. The Distribution of the Lognormal -- Normal Increments

As a special case of the subordinate distributions in the last section, consider a process $X(t)$ whose independent increments $\Delta X(t)$ are normally distributed, directed by a process $T(t)$, whose independent increments are lognormally distributed. By a lognormal distribution, we mean a random variable $x$ whose density is:

$$
f\left(x ; \mu, \sigma_{1}^{2}\right)=\frac{1}{2 \pi \sigma_{1}^{2} \cdot x} e^{-\frac{(\log x-\mu)^{2}}{2 \sigma_{1}^{2}}}
$$

It is named the lognormal distribution because $u=\log x$ is normally distributed with mean $\mu$ and variance $\sigma_{1}{ }^{2} .^{16}$ As may be easily shown, the mean of $x$ is $\mu_{x}=e^{\mu+\sigma_{1}{ }^{3} / 2}$ and the variance of $x$ is

$$
\sigma_{x}^{2}=e^{2 \mu+\sigma_{1}^{2}}\left[e^{\sigma_{1}^{2}}-1\right]
$$

Theorem 4 now tells us that if $\Delta X(t)$ is distributed normally with mean 0 and variance $\sigma_{2}{ }^{2}$, the increments $\Delta X(T(t))$ of the lognormal - normal process have mean 0 and variance:

16. Aitchison and Brown [1] have a complete discussion of the properties of the lognormal distribution. 


$$
\sigma_{\Delta X(T(t))}^{2}=\sigma_{2}^{2} \cdot e^{\mu+\sigma_{1}^{2 / 3}}
$$

Corollary 4.1 says that for given $\left(\mu+\sigma_{1}^{3 / 2}\right)$ but increasing $\sigma_{1}{ }^{3}$, the variance of this distribution stays constant while its kurtosis increases as much as desired. Presumably this distribution will fit the observed distribution of cotton price changes much better than the normal distribution, and might do better than the stable distributions. ${ }^{17}$

Theorem 5 A random process subordinated to a normal process with independent increments distributed $\mathrm{N}\left(0, \sigma_{\mathrm{a}}{ }^{2}\right)$ and directed by a $10 \mathrm{~g}$ normal with independent increments (and parameters $\mu$ and $\sigma_{1}{ }^{2}$ ) has the following lognormal - normal increments:
(4) $f_{L N N}(y)=\frac{1}{2 \pi \sigma_{1}^{2} \cdot \sigma_{9}^{2}} \int_{0}^{\infty} v^{-3 / 2} \cdot e^{-\frac{(10 g v-\mu)^{2}}{2 \sigma_{1}{ }^{2}}} \cdot e^{-\frac{y^{2}}{2 v \sigma_{2}{ }^{2}} d v}$

Proof: As in Theorem 4, the distribution of $\Delta \mathrm{X}(\mathrm{T}(\mathrm{t}))$ is just the expectation of the distribution of $F_{x}\left(0, t \sigma_{z}^{2}\right)$, the expectation being taken over the increments of $T(t)$. That is:

$$
f_{L N N}(y)=E_{t}\left[f_{N}\left(0, t \sigma_{z}^{a}\right)(y)\right]
$$

where $t$ is lognormally distributed. Thus

$$
f_{L N N}(y)=\int_{0}^{\infty}\left(\frac{1}{2 \pi v \sigma_{3}^{2}} e^{-\frac{y^{2}}{2 v \sigma_{3}^{2}}} \mid\left(\frac{1}{2 \pi \sigma_{1}^{2} \cdot v} e^{-\frac{(\log v-\mu)^{2}}{2 \sigma_{1}}}\right) d v\right.
$$

Simplification yields formula (4).

This relatively complicated formula may be approximated by numerical integration techniques.

17. In fact, the lognormal-normal distribution was included because it was found empirically to be the most useful. See Clark [6] for other distributions subordinate to the normal. It is not clear theoretically why operational time should be lognormally distributed. 
III. TESTS OF THE CONDITIONAL DISTRIBUTION

$[\Delta \mathrm{X}(\mathrm{T}(\mathrm{t})) \mid \Delta \mathrm{T}(\mathrm{t})]$ AND THE DISTRIBUTION OF $\Delta \mathrm{T}(\mathrm{t})$

At the end of Section I, it was mentioned that the price process, $X(T(t))$, evolved at different rates on different days. An obvious measure of this speed of evolution is trading volume. In fact, if the price changes on individual trades were uncorrelated, $T(t)$, the directing process, would be the cumulative trading volume up to time $t$. The distribution of the increments of the price process $\Delta \mathrm{X}(\mathrm{T}(t))$ would then have a distribution subordinate to that of the price changes on individual trades, and directed by the distribution of trading volume.

The way to test the hypothesis that trading volume in some sense measures the speed of evolution is clear; the relationship between trading volume and price change variance must be examined. If trading volume is not related to the speed of evolution, there should be no correlation between $V(t)$ (trading volume on day $t$ ) and $[\Delta X(T(t))]^{2}$. If trading volume is the directing process, the relationship should be linear, with the proportionality coefficient representing the variance of $\Delta X(t)$.

The first approach was the grouping of the two samples of 1000 observations each on cotton futures prices into 20 groups of 50 each by increasing volume. ${ }^{18}$ The sample variance and kurtosis within each group were calculated. These results displayed in Table 1. Trading volume and price change variance seem to have a curvilinear relationship.

More significantly, note that the kurtosis has been very much reduced when price changes with similar volumes are considered. The variance of the sample kurtosis from a normal population is $24 / \mathrm{n}$, where $n$ is the

18. See the Appendix for a description of the data used. 


\section{Table 1}

\section{Price Change Variance and}

\section{Kurtosis by Volume Class}

A. Sample One: January 17, 1947, to August 31, 1950

\begin{tabular}{|c|c|c|c|c|c|}
\hline \multirow{22}{*}{$\begin{array}{l}\text { Entire) } \\
\text { Sample) }\end{array}$} & \multicolumn{2}{|c|}{ Volume Range } & $\begin{array}{c}\text { Volume } \\
\text { Mean }\end{array}$ & $\begin{array}{c}\text { Sample } \\
\text { Variance }\end{array}$ & $\begin{array}{c}\text { Sample } \\
\text { Kurtosis }\end{array}$ \\
\hline & 326 & -12156 & 2718.94 & 584.55 & 19.45 \\
\hline & 326 & 939 & 718.4 & 30.51 & 3.95 \\
\hline & 948 & -1123 & 1030.7 & 59.60 & 3.53 \\
\hline & 1124 & - $\quad 1297$ & 1223.7 & 48.87 & 4.64 \\
\hline & 1298 & -1434 & 1371.0 & 102.70 & 5.09 \\
\hline & 1435 & -1556 & 1493.2 & 105.46 & 4.02 \\
\hline & 1558 & $-\quad 1710$ & 1628.7 & 73.76 & 3.21 \\
\hline & 1711 & - 1873 & 1788.4 & 104.65 & 2.73 \\
\hline & 1874 & 2032 & 1955.3 & 138.53 & 4.75 \\
\hline & 2033 & -2225 & 2121.8 & 173.38 & 4.18 \\
\hline & 2227 & 2408 & 2316.2 & 300.17 & 4.55 \\
\hline & 2408 & - 2595 & 2504.9 & 310.99 & 2.93 \\
\hline & 2608 & - $\quad 2807$ & 2712.4 & 240.52 & 3.47 \\
\hline & 2807 & 2995 & 2912.5 & 347.13 & 3.01 \\
\hline & 2998 & - 3279 & 3146.0 & 486.91 & 4.55 \\
\hline & 3284 & 3539 & 3399.6 & 352.68 & 2.95 \\
\hline & 3540 & $-\quad 3800$ & 3676.2 & 800.07 & 2.61 \\
\hline & 3803 & 4194 & 4013.8 & 711.67 & 3.14 \\
\hline & 4204 & 4737 & 4434.3 & 785.06 & 2.07 \\
\hline & 4739 & - 5512 & 5149.3 & 2716.77 & 5.14 \\
\hline & 5556 & -12156 & 6782.3 & 3695.87 & 6.35 \\
\hline
\end{tabular}


Sample Two: March 24, 1951, to February 10, 1955

\begin{tabular}{|c|c|c|c|c|c|}
\hline \multirow{3}{*}{$\begin{array}{l}\text { Entire) } \\
\text { Sample) }\end{array}$} & \multicolumn{2}{|c|}{ Volume Range } & \multirow{2}{*}{$\begin{array}{c}\begin{array}{c}\text { Volume } \\
\text { Mean }\end{array} \\
2733.40\end{array}$} & \multirow{2}{*}{$\begin{array}{c}\begin{array}{c}\text { Sample } \\
\text { Variance }\end{array} \\
501.73\end{array}$} & \multirow{2}{*}{$\begin{array}{c}\begin{array}{c}\text { Sample } \\
\text { Kurtosis }\end{array} \\
20.49\end{array}$} \\
\hline & 488 & -10571 & & & \\
\hline & 488 & 979 & 794.7 & 18.67 & 2.64 \\
\hline & 985 & $-\quad 1187$ & 1078.4 & 42.93 & 3.57 \\
\hline & 1202 & -1336 & 1271.4 & 56.56 & 3.03 \\
\hline & 1337 & -1509 & 1432.2 & 59.52 & 2.73 \\
\hline & 1510 & $-\quad 1631$ & 1570.0 & 53.44 & 2.65 \\
\hline & 1634 & -1766 & 1699.1 & 87.41 & 2.30 \\
\hline & 1768 & - 1895 & 1822.5 & 89.84 & 4.04 \\
\hline & 1899 & 2026 & 1963.8 & 105.71 & 3.71 \\
\hline & 2029 & $-\quad 2190$ & 2110.8 & 136.25 & 3.59 \\
\hline & 2191 & -2353 & 2266.1 & 178.86 & 3.57 \\
\hline & 2355 & - 2537 & 2445.7 & 214.31 & 4.82 \\
\hline & 2538 & $-\quad 2705$ & 2615.1 & 223.56 & 2.68 \\
\hline & 2709 & - 2913 & 2816.6 & 283.70 & 4.82 \\
\hline & 2913 & $-\quad 3179$ & 3043.7 & 263.73 & 2.12 \\
\hline & 3180 & $-\quad 3434$ & 3294.6 & 295.70 & 3.63 \\
\hline & 3436 & $-\quad 3763$ & 3581.1 & 520.92 & 2.98 \\
\hline & 3765 & $-\quad 4128$ & 3935.4 & 642.46 & 1.99 \\
\hline & 4160 & -4795 & 4509.6 & 937.33 & 2.35 \\
\hline & 4800 & $-\quad 5754$ & 5238.9 & 2067.90 & 8.76 \\
\hline & 5759 & -10571 & 7178.2 & 3659.21 & 4.41 \\
\hline
\end{tabular}


sample size. Thus any sample kurtosis that lies between 1.6 and 4.4 are within 2 standard deviations of the true value, 3 , expected with a normal parent. The vast majority of sample kurtoses lie within this range for both samples.

The average sample kurtosis is larger than 3 for both samples; but this is just as expected. Each volume class contains a range of volumes; just as in the case of the entire sample, this makes the sample distribution of the daily price changes non-normal. However, since each volume class contains a much smaller range of volumes, this phenomenon is considerably reduced. Note that the last two volume classes in both samples have significantly higher kurtosis than the other classes; this is clearly caused by the larger range of volumes included in these classes. Grouping by volume classes has brought the kurtosis of the price change distribution to within two standard deviations of that expected from a normal parent, while the original kurtosis of the whole sample is 100 standard deviations away.

To investigate the curvilinear relationship between price variance and trading volume, the regressions in Table 2 were used. The results indicate that either $\sigma^{2}=A e^{\alpha v}$ or $\sigma^{2}=\mathrm{Bv}^{\beta}$ are equally good in explaining movement of price variance. The first faces the theoretical objection that $\sigma^{2}(0) \neq 0$ but is negligibly different from 0 for the sizes of numbers we are using. The linear specification is clearly worse; the $F$ statistics calculated for linear regressions with a constant term were about 125 , with a large negative intercept. The high negative intercept term for the unconstrained regressions indicates that 


\section{Table 2}

\section{Daily Price Change Variance as a Function of Daily Volume}

Sample No. 1:
a) $\log \left(\Delta x^{2}\right)=\frac{1.977+.0008219 v}{(.149)(.00004784)}$
$F=295.20$
b) $\log \left(\Delta \mathrm{X}^{2}\right)=-12.71+2.181 \log (\mathrm{v})$
$F=280.38$
c)
$\Delta \mathrm{X}^{2}=\begin{aligned} & .31374 \mathrm{v} \\ & (.02428)\end{aligned}$
$F=156.25$

Sample No. 2:
a) $\log \left(\Delta \mathrm{X}^{2}\right)=1.968+.0007334 \mathrm{v}$
$(.149)(.00004766)$
$F=236.83$
b) $\log \left(\Delta x^{2}\right)=\frac{-12.73+2.1503}{(1.05)(.1350)} \log (v)$
$F=253.69$
c)

$$
\Delta \mathrm{X}^{2}=\frac{.25598 \mathrm{v}}{(.02611)}
$$
$F=86.24$

(The numbers in parentheses are standard errors)

$$
F_{.01}(1,998)=F_{.01}(1,999)=6.66
$$


the linear model performs very poorly, as does the low F-statistic. Even if all trades on any given day are perfectly correlated, the dependence of price change variance on volume would be only $\sigma^{2} \sim v^{2}$; the data reject even this high dependence as too low.

To see how this curvilinear dependence of price change variance on volume might occur, consider how the futures market actually works. At any time there are a number of traders in the market who have expectations about the price of a given cotton contract. Some will have long positions (holding contracts), some will have short positions (having sold contracts), and some may have no net position at all if they are waiting for more favorable conditions. When new information (in the form of data that the traders consider relevant) flows to the market, both prices and traders' price expectations will change. If the information is uncertain (i.e., some traders shift expectations up and others down on the basis of the information), or if only "inside" traders get the information first, then large price changes will be coincident with high volumes. On the other hand, very large price changes will probably be due to information that is perceived by all traders to move the price in one direction. News of widespread insect problems might be an example of this sort of information in the cotton futures market. In this case, all traders would revise their expectations in the same direction, and the price change would have relatively low volume.

Thus the relationship $\operatorname{Var}(\Delta X)=C v^{2} \cdot 18$ is seen as combination of correlation of price changes on individual trades, and a deficiency of volume at high price changes, caused by traders moving their expectations 
in unison. Trading volume is taken as an instrument for the true operational time, or an "imperfect clock" measuring the speed of evolution of the price change process. The regressions in Table 2 are taken as the way to adjust the "volume clock" to get the best obtainable estimate of operational time.

It is now natural to use these equations estimating "operational time" to adjust price changes and find the distribution of $\Delta \mathrm{X}(t)$. That is, if $\Delta T(t)=f(v(t))$, then $\Delta X(T(t)) / \sqrt{f(v(t))}$ should be distributed as $\Delta \mathrm{X}(\mathrm{t})$. The results of this adjustment are summarized in Table 3 . The distribution of the Kolmogorov-Smirnov statistic under a complex null hypothesis is not known exactly, but the significance level is reduced in the normal case. Although these $K-S$ statistics are very encouraging, the sample kurtosis is still too high. This is attributable to one of two causes. Either $\Delta T(t)=f(v(t))$ and the estimation procedure in Section II has not found the true $f(v)$, or $\Delta T(t)=f(v(t)) \cdot u(t)$ where $u(t) \neq 1$. In either case the transformation $\Delta x(t) / \sqrt{f(v(t))}$ leaves a small deterministic or random element of operational time still in the adjusted series. Since the results in section II tell us that introduction of operational time will always lead to increased kurtosis, either type of error should lead to results like those in Table $3 .^{19}$ The relative strength of these numbers is seen when the figures in Table 3 are compared

19. Although the introduction of operational time always increases kurtosis, it is easy to think of a stistical adjustment procedure that could make $k<3$. By making overestimates of variance (or $f(v)$ ) on very high price changes, but not having them too low on small price changes, it is possible to cut of $f$ the tails entirely in the adjusted distribution. Any regression method of estimating will usually have both positive and negative errors on the low and high ends of the volume range. In fact, $f(v)$ in Table 2 tends to underestimate on the high end. 
$\underline{\text { Table } 3}$

Distribution of $\frac{\Delta X(t)}{\sqrt{f(v(t))}}$ for $f(v)=A v^{\alpha}$ and $f(v)=B e^{\beta v}$

\begin{tabular}{|c|c|c|}
\hline & Sample \#1 & Sample 非2 \\
\hline$f(v)$ & $e^{-12.71} v^{2.1818}$ & $e^{-12.73} v^{2.1503}$ \\
\hline $\begin{array}{l}\text { K-S test against } \\
\text { normality }\end{array}$ & $.0321(.26)^{20}$ & $.0195(.84)$ \\
\hline Kurtosis & 4.55 & 4.56 \\
\hline$f(v)$ & $\mathrm{e}^{1.977} \mathrm{e}^{.0008219 \mathrm{v}}$ & $e^{1.968} e^{.0007334 v}$ \\
\hline $\begin{array}{l}\text { K-S test against } \\
\text { normality }\end{array}$ & $.0294(.35)$ & $.0239(.62)$ \\
\hline Kurtosis & 4.26 & 4.18 \\
\hline
\end{tabular}

20. The numbers in parentheses indicate the probability that the preceding value of the K-S statistic will be exceeded when the null hypotheses is true. 
with those in Table 1 for the entire sample. The K-S test statistics against normality are .114 for Sample No. 1 and .121 for Sample No. 2, both of which have probability of less than .000001 of occurrence if the sample is drawn from the normal parent. Similarly, the kurtosis has been reduced a very significant amount.

Although the results are far from perfect, they are good enough to conclude that the "imperfect clock" hypothesis is a good approximation to the truth at this level of analysis.

There is, then, a strong case for normality of price change when it is adjusted for operational time. To find the distribution of price change, however, the distribution of $\Delta T(t)$ must be found. Both the Gamma and Lognormal distributions were fit to the two specifications of $\Delta T(t)=(f(v(t))$ for operational time. Only the Lognormal results are reported in Table 4, since the Lognormal fit very much better than the Gamma.

The easiest way to test for lognormality is to take logarithms of the sample and test for normality; since this is the method used, the kurtosis of the sample after logarithms have been taken is also displayed in Table 4. Note that testing the lognormality of $f(v)=A v^{\alpha}$ is equivalent to testing the lognormality of $v$, while testing the lognormality of $f(\dot{v})=B e^{\beta v_{i s}}$ equivalent to testing the normality of $v$. The results in Table 4 indicate that $v$ is lognormally distributed as opposed to normally distributed; the model $f(v)=A v^{\alpha}$ is the better one to use, given both models seem to work equally well as operational time. 
Table 4

Tests of Lognormality of $\Delta T(t)=f(v(t))$ for $f(v)=A v^{\alpha}$ and $f(v)=B e^{\beta v}$

\begin{tabular}{|c|c|c|}
\hline & Sample 非 & Sample 非2 \\
\hline$E(v)$ & $e^{-12.71} v^{2.1818}$ & $\mathrm{e}^{-12.73} \mathrm{v}^{2.1503}$ \\
\hline $\begin{array}{l}\text { K-S statistic } \\
\text { against } \\
\text { lognormality }\end{array}$ & $.03343(.21)^{21}$ & $.01562(.97)$ \\
\hline $\begin{array}{l}\text { Kurtosis of } \\
\log (f(v))\end{array}$ & 2.929 & 2.858 \\
\hline$f(v)$ & $\mathrm{e}^{1.977} \mathrm{e}^{.0008219 \mathrm{v}}$ & $\mathrm{e}^{1.968} \mathrm{e}^{.0007334 \mathrm{v}}$ \\
\hline $\begin{array}{l}\text { K-S statistic } \\
\text { against } \\
\text { lognormality }\end{array}$ & $.08303(.00001)$ & $.1114(.00000)$ \\
\hline $\begin{array}{l}\text { Kurtosis of } \\
\log (f(v))\end{array}$ & 6.744 & 6.115 \\
\hline
\end{tabular}

21. Again numbers in parentheses are the probability of exceeding the given K-S statistic if the null hypothesis were true. 
All of the results above are very strong evidence in favor of the finite-variance subordination model. They also point out that the marginal distribution (unconditional on operational time) of price changes should be lognormal-normal rather than stable. If $\Delta \mathrm{X}(t)$ is normal, then $\Delta \mathrm{T}(t)$ must have a stable distribution with a very long tall (no finite mean) in order that $\Delta X(T(t))$ have a stable distribution. If this were the case, the lognormal fit in Table 4 should be much worse.

IV. A DIRECT TEST OF THE TWO COMPETING HYPOTHESES

Two approaches were used to test the Lognormal-Normal (LN) family against the stable (S) family of distributions as the parent of the observed distribution of price changes for cotton futures.

The first test was a Bayesian one, with discrete prior and posterior distributions over the two hypotheses. The construction of this test, although not completely rigorous, was well-motivated by practical considerations. Suppose a decision-maker is trying to decide whether cotton futures price changes have a stable or lognormal-normal distribution, and his initial position before examination of the data is complete ignorance. Then his prior distribution should have $P(S)=P(L N)=.5$, and presumably after the sample information has been examined, these probabilities will change.

Calculation of posterior probabilities could proceed in straightforward fashion if $S$ and $L N$ were not complicated, with an infinite number of parameter values available within each hypothesis. In view of the fact that analytical calculation of likelihoods as functions of parameter values was considered impossible by the author, a second-best approach was used. Twenty-five 
simple hypotheses (that is, exactly-specified sets of parameters) within each set of $S$ and $L N$ were chosen, using all the prior information possible about the region in which these parameter points should lie. Such a strategy assumes that the likelihood functions are smooth and do not have high peaks between the selected points in parameter space. It also makes use of the present decision-maker's relative indifference about the exact parameter values. Once this method is adopted, prior probabilities $\left\{P_{1}^{A}\right\}_{1=1}^{50}$ may be assigned in such a way that $P_{1}^{A}$ are the same for all $i$, and that $\sum_{S} P_{1}{ }^{A}=\sum_{L N} P_{1}{ }^{A}=.5$. Posterior probabilities $\left\{P_{1}{ }^{B}\right\}_{1=1}^{50}$ for these hypotheses may be calculated using Bayes' rule:

$$
P_{1}^{B}=\frac{P_{1}^{A} \cdot L\left(S_{1} \mid H_{1}\right)}{\sum_{1} L\left(S_{1} \mid H_{1}\right) P_{1}^{A}}
$$

where $L\left(S_{w} \mid H_{1}\right)$ is the likelihood of the sample given hypothesis $i$. Posterior probabilities for $S$ and $L N$ are $\sum_{S} P_{1}^{B}$ and $\underset{L N}{\Sigma} P_{1}^{B}$ respectively. Each simple hypothesis was given a prior probability of 0.02. The parameters which constituted each simple hypothesis were made up by using theoretical considerations to guess what combinations of parameters would maximize the likelihood of the sample:

\section{Lognormal-Normal distributions:}

Let $\Delta T(t)=T(t)-T(t-1)$ be distributed lognormally with parameters $\mu, \sigma_{1}{ }^{2}$. That is, $\log (\Delta T(t))$ is distributed normally with mean $\mu$ and variance $\sigma_{1}^{2}$. Let $\Delta X(t)=X(t)-X(t-1)$ be distributed normally with mean 0 
and variance $\sigma_{3}{ }^{2} \cdot F_{T}$ has mean $e^{\mu+\sigma_{1}^{2} / 2}$ and variance $e^{2 \mu+\sigma_{1}^{2}}\left(e^{\sigma_{1}^{2}}-1\right)$. Theorem 4 then says that the variance of the distribution of $\Delta \mathrm{X}(\mathrm{T}(\mathrm{t}))=$ $X(T(t))-X(T(t-1))$ is $\sigma_{2}^{2} \cdot e^{\mu+\sigma_{1}^{2} / 2}$

Since the data are normalized so that the sample variance is 1 , one constraint on the parameters for the prior distribution of the lognormalnormal is:

$$
\sigma_{8}^{2} \cdot e^{\mu+\sigma_{1}^{2} / 2}=1
$$

Corollary 4.1 states that the kurtosis of the lognormal-normal family is

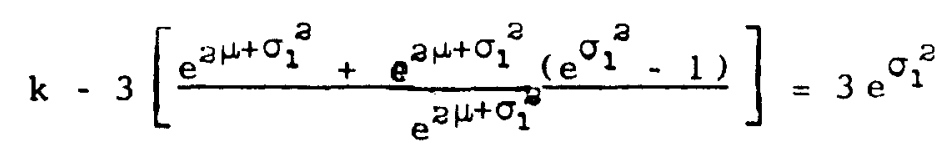

Thus another constraint on the parameters in the prior distribution is $\sigma_{1}^{2}=\log (k / 3)$ where $k$ is the sample kurtosis.

\section{Stable distributions:}

The characteristic function for this family is $e^{-\gamma|u|^{\alpha}}$, which converges to $e^{-\sigma^{2} u^{2} / 2}$ as $\alpha \rightarrow 2$, when the normal distribution is obtained. If $\alpha<2$, then $\gamma$ must be smaller than 0.5 to fit a sample with a sample variance of 1 , so $1<\alpha<2$ and $0<\gamma<.5$ is the correct region for the prior. Since these restrictions represent far less information than the restrictions on the lognormal-normal, some preliminary calculations of the likelihood were made to narrow down the region for the prior which would present the stable distributions in the most favorable light.

The concepts involved in constructing this test are elementary; the difficult problems are the practical ones. In order to calculate the likelihood of a sample, the density of the distribution of the sample under the null hypothesis must be known. For the case at hand, these densities are: 
(5) Stable: $\mathrm{f}_{\mathrm{S}}(\mathrm{x} ; \alpha, \gamma)=1 / \pi \int_{0}^{\infty} \cos (\mathrm{ux}) \mathrm{e}^{-\gamma|u|^{\alpha}} \mathrm{du}$

(6) Lognormal-Normal:

$$
f_{L N}\left(x ; \mu, \sigma_{1}^{2}, \sigma_{z}^{2}\right)=\frac{1}{2 \pi \sigma_{1}^{2} \sigma_{2}^{2}} \int_{0}^{\infty} v^{-3 / 2} e^{-\frac{(\log v-\mu)^{2}}{2 \sigma_{1}^{2}}-\frac{x^{2}}{2 v \sigma^{2}}} d v
$$

Expression (5) is a consequence of the fact that the characteristic function of a distribution is its Fourier transform, while expression (6) is the lognormal-normal density derived in II. Since neither of the integrals on the right hand side of these equations may be solved analytically, the problem of finding an approximate likelihood function for any sample reduces to finding values of $f_{S}$ or $f_{L N}$ for many different values of $x$, and then interpolating to find the likelihoods for sample values of $x$.

The density for the stable distributions (5) is by far the easier to approximate accurately; the integral on the right is the Fourier transform of $e^{-\gamma|u|^{\alpha}}$, and the fast Fourier transform methods that have been recently developed ${ }^{22}$ are extremely accurate. For the test at hand, the value of $f_{S}(x)$ was tabulated for $x \in[0,10]$ at 2048 equal intervals. This range was adequate, for no observations were recorded at more than 10 sample standard deviations from 0 .

Calculation of $f_{L N}(x)$ was less accurate and more costly. First the interval over which the integrand in (6) was greater than $10^{-1 b}$ was found. The required integral was then calculated by using Simpson's rule with 601

22. Cooley, J. W., and J. W. Tukey, [7]. In fact, these methods are accurate and fast enough to calculate 4 or 5 place tables of the stable distributions at relatively small expense, if anyone so desired. 
points after dividing the interval up so that the interpolation points would be closer together when the integrand was changing rapidly. This process was repeated for $x \in[0,10]$ at 101 points. Both distributions required small adjustments so that their numerical integral on $[-10,10]$ equalled 1 .

Tables 5 and 6 give posterior probabilities and likelihoods for the samples, given the parameters in the distributions. Again, all prior probabilities are 0.02 .

The posterior probability of $S$ and $L N$ for the two samples are: Sample 1: $\quad P(S)=.11 \times 10^{-5} \quad P(L N)=.999999$, Sample 2: $P(S)=.0007 \quad P(L N)=.9993$. These results are very convincing evidence that the observed leptokurticity in the price change distribution for cotton futures is caused by the fact that the data are recorded in "clock" time rather than operational time.

Note that the results are independent of the choice of prior distribution as long as at least one of the prior points in the LN hypothesis is in the region of high likelihood. This is only another way of saying that the likelihood of a lognormal-normal having generated the sample is very significantly higher than the likellhood for any stable distributions.

One way to see why this is true is to look at the maximum likelihood distributions in each family, as displayed in Table 7. The primary difference is that the lognormal-normal distribution is larger at 0 and smaller in the tails; if there were very many observations 8 or 9 sample 
$\underline{\text { Table } 5 \mathrm{~A}}$

Posterior Probabilities and Likelihoods of Sample 1

Stable Distribution

\begin{tabular}{|c|c|c|c|c|c|}
\hline \multirow[b]{2}{*}{$\alpha$} & \multirow[b]{2}{*}{$Y$} & \multicolumn{2}{|c|}{$\begin{array}{c}\text { Likelihood }= \\
\qquad A \times 10^{-B}\end{array}$} & \multicolumn{2}{|c|}{$\begin{array}{c}\text { Posterior Prob. } \\
\mathrm{C} \times 10^{-\mathrm{D}}\end{array}$} \\
\hline & & $\mathrm{A}$ & $\mathrm{B}$ & $\mathrm{C}$ & D \\
\hline 1.3 & .3 & .7028 & 539 & .6895 & 8 \\
\hline 1.3 & .325 & .8671 & 539 & .8507 & 8 \\
\hline 1.35 & .275 & .4910 & 539 & .4817 & 8 \\
\hline 1.35 & .3 & .5742 & 538 & .5634 & 7 \\
\hline 1.35 & .325 & .3453 & 538 & .3388 & 7 \\
\hline 1.35 & .35 & .2057 & 539 & .2018 & 8 \\
\hline 1.375 & .287 & .5393 & 538 & .5291 & 7 \\
\hline 1.375 & .312 & .9776 & 538 & .9788 & 7 \\
\hline 1.375 & .337 & .1378 & 538 & .1352 & 7 \\
\hline 1.4 & .275 & .2630 & 538 & .2580 & 7 \\
\hline 1.4 & .3 & .1476 & 537 & .1449 & 6 \\
\hline 1.4 & .325 & .5001 & 538 & .4907 & 7 \\
\hline 1.4 & .35 & .1913 & 539 & .1877 & 8 \\
\hline 1.425 & .287 & .1139 & 537 & .1117 & 6 \\
\hline 1.425 & .312 & .1156 & 537 & .1134 & 6 \\
\hline 1.425 & .337 & .1045 & 538 & .1025 & 7 \\
\hline 1.45 & .275 & .4439 & 538 & .4355 & 7 \\
\hline 1.45 & .30 & .1397 & 537 & .1371 & 6 \\
\hline 1.45 & .325 & .3047 & 538 & .2989 & 7 \\
\hline 1.475 & .287 & .8759 & 538 & .8594 & 7 \\
\hline 1.475 & .312 & .5729 & 538 & .5621 & 7 \\
\hline 1.475 & .337 & .3739 & 539 & .3668 & 8 \\
\hline 1.5 & .275 & .2698 & 538 & .2647 & 7 \\
\hline 1.5 & .3 & .5507 & 538 & .5403 & 7 \\
\hline 1.55 & .275 & .6532 & 539 & .6409 & 8 \\
\hline
\end{tabular}




\section{Table 5B}

Posterior Probabilities and Likelihoods for Sample 1

Lognormal - Normal Distribution

$\begin{array}{cc}\text { Likelihood }= & \text { Posterior Prob. }= \\ \mathrm{A} \times 10^{-\mathrm{B}} & \mathrm{C} \times 10^{-\mathrm{D}}\end{array}$

\begin{tabular}{ccccccc}
$\mu$ & $\sigma_{1}^{2}$ & $\sigma_{2}^{2}$ & $\mathrm{~A}$ & $\mathrm{~B}$ & $\mathrm{C}$ & $\mathrm{D}$ \\
\hline-.5 & 1.5 & .7 & .3677 & 532 & .3608 & 1 \\
-.5 & 1.5 & .25 & .3425 & 537 & .3362 & 6 \\
-.5 & 2.5 & .5 & .2418 & 533 & .3272 & 2 \\
-1.49 & 1.6 & 2.0 & .5673 & 537 & .5566 & 6 \\
-1.21 & 1.6 & 1.5 & .2124 & 534 & .2084 & 3 \\
-.8 & 1.6 & 1.0 & .9510 & 533 & .9389 & 2 \\
-.11 & 1.6 & .5 & .4191 & 532 & .4112 & 1 \\
.59 & 1.6 & .25 & .3236 & 532 & .3175 & 1 \\
-1.31 & 1.8 & 1.5 & .6728 & 534 & .6601 & 3 \\
-.90 & 1.8 & 1.0 & .3198 & 532 & .3138 & 1 \\
-.21 & 1.8 & .5 & .1047 & 531 & .1026 & 0 \\
.49 & 1.8 & .25 & .7619 & 532 & .7475 & 1 \\
-1.59 & 2.0 & 2.0 & .1591 & 537 & .1473 & 6 \\
-1.41 & 2.0 & 1.5 & .1254 & 533 & .1230 & 2 \\
-1.0 & 2.0 & 1.0 & .4798 & 532 & .4707 & 1 \\
-.31 & 2.0 & .5 & .6633 & 532 & .6508 & 1 \\
.39 & 2.0 & .25 & .2920 & 532 & .2865 & 1 \\
.30 & 1.8 & .30 & .1084 & 531 & .1064 & 0 \\
.13 & 1.8 & .35 & .8771 & 532 & .8605 & 1 \\
.13 & 1.85 & .35 & .8512 & 532 & .8356 & 1 \\
.15 & 1.85 & .35 & .8434 & 532 & .8275 & 1 \\
.11 & 1.85 & .35 & .7978 & 532 & .7827 & 1 \\
-1.10 & 2.0 & 1.10 & .3919 & 532 & .3845 & 1 \\
-1.0 & 2.2 & .9 & .5261 & 532 & .5162 & 1 \\
.9 & 2.0 & 1.10 & .6026 & 534 & .5912 & 3
\end{tabular}


Table 6A

Posterior Probabilities and Likelihoods for Sample 2

Stable Distribution

Likelihood $=$ $\mathrm{Ax} 10^{-\mathrm{B}}$
Posterior Prob. = $\mathrm{C} \times 10^{-\mathrm{D}}$

\begin{tabular}{|c|c|c|c|c|c|}
\hline$\alpha$ & $Y$ & A & $\mathrm{B}$ & $\mathrm{C}$ & $\mathrm{D}$ \\
\hline 1.35 & .275 & .1806 & 520 & .1617 & 4 \\
\hline 1.35 & .3 & .2104 & 520 & .1886 & 4 \\
\hline 1.40 & .275 & .5946 & 520 & .5329 & 4 \\
\hline 1.40 & .3 & .2945 & 520 & .2640 & 4 \\
\hline 1.45 & .275 & .5909 & 520 & .5296 & 4 \\
\hline 1.45 & .3 & .1407 & 520 & .1261 & 4 \\
\hline 1.50 & .275 & .1877 & 520 & .1682 & 4 \\
\hline 1.45 & .25 & .8906 & 521 & .7982 & 5 \\
\hline 1.475 & .262 & .2776 & 520 & .2488 & 4 \\
\hline 1.35 & .287 & .2919 & 520 & .2616 & 4 \\
\hline 1.375 & .262 & .1151 & 520 & .1032 & 4 \\
\hline 1.375 & .275 & . 3889 & 520 & .3486 & 4 \\
\hline 1.375 & .287 & .4973 & 520 & .4457 & 4 \\
\hline 1.375 & .3 & .2881 & 520 & .2582 & 4 \\
\hline 1.375 & .312 & .9115 & 521 & .8170 & 5 \\
\hline 1.40 & .262 & .2303 & 520 & .2063 & 4 \\
\hline 1.40 & .287 & .6217 & 520 & .2346 & 4 \\
\hline 1.425 & .262 & .3374 & 520 & .3024 & 4 \\
\hline 1.425 & .275 & .6861 & 520 & .6149 & 4 \\
\hline 1.425 & .287 & .5783 & 520 & .5138 & 4 \\
\hline 1.425 & .3 & .2255 & 520 & .2021 & 4 \\
\hline 1.45 & .262 & .3558 & 520 & .3189 & 4 \\
\hline 1.45 & .287 & .4246 & 520 & .3815 & 4 \\
\hline 1.475 & .275 & .3756 & 520 & . 3366 & 4 \\
\hline 1.475 & .287 & .2316 & 520 & .2076 & 4 \\
\hline
\end{tabular}


Table 6B

Posterior Probabilities and Likelihoods for Sample 2

Lognormal - Normal Distribution

Likelihood = A $\times 10^{-B}$
Posterior Prob. = $\mathrm{C} \times 10^{-\mathrm{D}}$

\begin{tabular}{|c|c|c|c|c|c|c|}
\hline$\mu$ & $\sigma_{1}^{2}$ & $\sigma_{2}^{2}$ & $A$ & B & $\mathrm{C}$ & D \\
\hline-.26 & 1.9 & .5 & .5415 & 517 & .4853 & 1 \\
\hline-1.05 & 1.9 & 1.0 & .6388 & 517 & .5726 & 1 \\
\hline-.36 & 1.9 & .5 & .6319 & 517 & .5664 & 1 \\
\hline-.21 & 1.8 & .5 & .3222 & 517 & .2888 & 1 \\
\hline-1.00 & 2.0 & 1.0 & .3048 & 517 & .2732 & 1 \\
\hline-.31 & 2.0 & .5 & .6611 & 517 & .5925 & 1 \\
\hline .39 & 2.0 & .25 & .3017 & 517 & .2704 & 1 \\
\hline-.21 & 2.0 & .5 & .1772 & 517 & .1588 & 1 \\
\hline .49 & 2.0 & .25 & .1086 & 517 & .9733 & 2 \\
\hline-.44 & 1.9 & .6 & .5520 & 517 & .4948 & 1 \\
\hline-.15 & 1.9 & .45 & .4847 & 517 & .4344 & 1 \\
\hline-.03 & 1.9 & .4 & .4376 & 517 & .3922 & 1 \\
\hline-.39 & 1.8 & .6 & .3013 & 517 & .2701 & 1 \\
\hline .02 & 1.8 & .4 & .2710 & 517 & .2429 & 1 \\
\hline .15 & 1.8 & .35 & .2713 & 517 & .2432 & 1 \\
\hline-.49 & 1.8 & .60 & .7456 & 517 & .6683 & 1 \\
\hline-.08 & 1.8 & .40 & .5358 & 517 & .4797 & 1 \\
\hline .05 & 1.8 & .35 & .4721 & 517 & .4231 & 1 \\
\hline-1.49 & 2.0 & .60 & .7656 & 517 & .6862 & 1 \\
\hline-.20 & 2.0 & .45 & .5707 & 517 & .5115 & 1 \\
\hline-.08 & 2.0 & .40 & .4897 & 517 & .4389 & 1 \\
\hline-.59 & 2.0 & .60 & .7588 & 517 & .6801 & 1 \\
\hline-.30 & 2.0 & .45 & .3874 & 517 & .3472 & 1 \\
\hline-.18 & 2.0 & .40 & .3035 & 517 & .2720 & 1 \\
\hline .05 & 1.9 & .40 & .1143 & 517 & .1024 & $I$ \\
\hline
\end{tabular}


$\underline{\text { Table } 7}$

Stable and Lognorma1-Norma1 Distributions which maximize the likelihood of the samples

$$
f_{\text {LNN }}(x) \quad f_{S}(x) \quad f_{L N N}(x) \quad f_{S}(x)
$$

$\mathrm{X}$

(1)
(2)

\section{Sample 1}

0

.1

.2

.3

.4

.5

.6

.7

.8

.9

1.0

1.2

1.4

1.6

1.8

2.0

2.5

3.0

3.5

4.0

4.5

5.0

6.0

7.0

8.0

9.0

10.0

.7755

.7356

.6394

.5302

.4340

.3557

.2925

.2414

.2000

.1665

.1393

.0989

.0716

.0526

.0393

.0298

.0156

$.870 \times 10^{-2}$

$.509 \times 10^{-2}$

$.310 \times 10^{-2}$

$.196 \times 10^{-2}$

$.127 \times 10^{-2}$

$.575 \times 10^{-3}$

$.282 \times 10^{-3}$

$.148 \times 10^{-3}$

$.815 \times 10^{-4}$

$.466 \times 10^{-4}$
(3)

(4)

Sample 2

.6856

.7717

.7159

.6711

.7399

.6999

.6304

.6566

.6549

.5696

.5495

.5882

.4972

.4449

.5092

.4216

.3569

.4275

.3494

.2880

.3503

.2849

.2349

.2822

.2300

.1935

.2249

.1849

.1606

.1786

.1487

.1342

.1420

.0882

.0951

.0822

.0605

.0688

.0556

.0431

.0507

.0392

.0318

.0380

.0287

.0277

.0289

.0217

.0129

.0153

.0114

$.891 \times 10^{-2} \quad .869 \times 10^{-2}$

$.720 \times 10^{-3}$

$.538 \times 10^{-2} \quad .517 \times 10^{-2}$

$.471 \times 10^{-2}$

$.388 \times 10^{-2} \quad .320 \times 10^{-2}$

$.339 \times 10^{-2}$

$.284 \times 10^{-2} \quad .206 \times 10^{-2}$

$.247 \times 10^{-2}$

$.221 \times 10^{-3} \quad .136 \times 10^{-2}$

$.191 \times 10^{-2}$

$.140 \times 10^{-3}$

$.637 \times 10^{-3}$

$.126 \times 10^{-2}$

$.954 \times 10^{-3}$

$.323 \times 10^{-3}$

$.820 \times 10^{-3}$

$.687 \times 10^{-3}$

$.175 \times 10^{-3}$

$.589 \times 10^{-3}$

$.515 \times 10^{-3}$

$.998 \times 10^{-4}$

$.440 \times 10^{-3}$

$.399 \times 10^{-3}$

$.598 \times 10^{-4}$

$.340 \times 10^{-3}$

(1) $\mu=.3 \sigma_{1}^{2}=1.8 \sigma_{2}^{2}=.3$

(2) $\alpha=1.4 \quad \gamma=.3$

(3) $\mu=-.49 \sigma_{1}^{2}=2.0 \sigma_{2}^{2}=.6$

(4) $\alpha=1.425 \quad \gamma=.275$ 
standard deviations from the mean, then the stable distribution would have fared much better on the tests. Instead, the sample is characterized by a few large observations that would be unlikely if the underlying distribution were normal, but not large enough to make the stable family a likely contender.

The evidence for the lognormal-normal is made stronger by the fact that the parameters derived from the theory for the prior distribution turn out to be the ones which maximize the likelihoods of both samples. Values which did not fit the restrictions gave much lower likelihoods for the samples.

On the other hand, the parameters of the stable distribution in the region of maximum likelihood were quite different from what was expected. Instead of $\alpha=1.8$ (or close to 2), $\alpha$ is much lower for both samples. This is a standard indication of specification error; the model is a bad approximation to the data, so the estimated parameters turn out to be different from the ones theoretical considerations indicate.

Estimates of $\alpha=1.4$ also cast doubt on the graphs of cumulative variance used by Mandelbrot. ${ }^{3}$ With an $\alpha$ this low, the sample variance as a function of sample size should have a pronounced upward slope. The relative flatness of these graphs indicates that the underlying population has high probabilities of large changes, but still a finite variance. The lognormal-normal distribution and other subordinate distributions are very suitable for representing this type of behavior.

23. Mandelbrot [14]. 
As second test of the two hypotheses, the Kolmogorov-Smirnov statistics testing each sample against the maximum likelihood distributions in Table 7 were calculated. The results are tabulated in Table 8 .

Note that the probabilities in parentheses are calculated using asymptotic results, but only a small number of steps is used for the numerical LNN distribution, so some "small sample" bias is involved.

Note also that the $\mathrm{K}-\mathrm{S}$ statistic is better at examining a distribution in the range of high density than in the tails; 20 out of 1000 observations at 20 standard deviations from the mean would change the likelihood results radically, but would have only a small effect on the $\mathrm{K}$-S results.

It is clear also that a bias in favor of the LNN hypothesis exists because of the statutory limits on price movements. However, examination of the data for 10 individual futures over the span 30 - 250 days until maturity ${ }^{24}$ revealed this bias to be extremely small. For all of these 10 futures, the limit ( 2 cents) was reached on only 3 occasions. Furthermore, the limit was an average of 8 sample standard deviations from 0 , thus making it a very weak constraint. It seems clear that the absence of this limitation would not have changed the above results.

In summary, then, the empirical evidence points to acceptance of the finite-variance subordination model. The standard Central Limit Theorem holds only when the number of random variables being added is constant (in probability limit, at least); in the case of speculative markets, this restriction is violated, and the limit distribution of price changes are subordinate to the normal distribution. 
Table 8

K-S statistics testing the samples against the maximum likelihood lognormal-normal and stable distributions:

Sample No. 1

LNN

$.0856(.80)$
Stable $.0374(.44)$
Sample No. 2

LNN Stable $.0955(.68) \quad .0438(.25)$

Numbers in parenthesis are the probability that the given value of the K-S statistic will be exceeded if the null hypothesis is true. 


\section{Appendix}

\section{Construction of a Long Time Series}

\section{for Cotton Futures}

The data on price, transactions, and volume for cotton futures is readily available in daily form for the years 1945-1958 in Trade in Cotton Futures. ${ }^{1}$ Considering the care with which the data were gathered, these daily figures potentially give very long and accurate time series. Except for a brief period during the Korean War when trading was suspended due to price controls, these series were recorded daily, and represent two periods of 1000 observations each. ${ }^{3}$ Such a wealth of data potentially prōvides idean circumstances for testing hypotheses about the structure of price movements on speculative markets.

As even the most casual observer of commodity markets knows, however, no contract (or future) has a lifetime that is this long. Contracts are made for delivery of cotton on a particular date (almost always on the fifteenth of March, May, July, October, or December). Trading in any particular contract begins about a year and a half before the delivery date on the contract, and ends on that date. Taken by itself, then, any one contract will yield a time series of only 300 points, many of which are taken when the market is thin and there is very little trading in that particular future (i.e., at the beginning and end of its life).

To remedy this situation, and generate longer time series that always

1. Trade in Cutton Futures, [19].

2. January 26, 1951 to March 23, 1951 .

3. "Sample No. 1" in the text is from January 17, 1947 to August 31, 1950, while "Sample No. 2" is from March 24, 1951 to February 10, 1955. 
represented prices and volumes on an active market, a continuous time series of prices and volume was constructed. The intent was to define a "contract" that matured a fixed distance in the future, analogous to "90 day futures" that exist in some foreign exchange markets. This fixed distance in the future was taken to be the average time to maturity of all futures in the market. Care was taken to make sure this was the same for all days, thereby avoiding the problem of changing the interest accruing to the seller of the contract.

The most straightforward way of defining this average future is to construct a weight function $h(\tau)$, where $T$ is the time distance from now that rach of the existing contracts mature. Since a few contracts usually come into existence a year and a half before the maturity date, this function was constructed for $T=1,2, \ldots 510$. Although the time pattern of futures contracts in existence clearly changes over time, the weight function applied to all dates should be the same, so that the "time to maturity" of the weighted average constructed remains relatively fixed in time. The "price" of this constructed cotton future is

$$
P_{t}=\frac{\sum_{T} W(T) P_{t}^{\top}}{\sum_{T} W(T)} \quad T=\{\text { set of all existing } T\}
$$

$\mathrm{P}_{\mathrm{t}}^{\top}=$ the price at $\mathrm{time} t$ of the contract maturing at time $t+\tau$. Typically, this sum includes eight terms.

To estimate $W(\tau)$, the average time pattern of contracts in existence, the proportion of all contracts was tabulated for all $T \in(0,510)$ days in the years 1946 to 1951. This consisted of about 25 proportions for each. These proportions were then averaged, giving the average proportion of open 
interest for each time distance in the future, for integral numbers of days. This procedure yields a rough approximation to the $W(\tau)$ function desired, except that the finite amount of data makes it possible for $W(\tau)$ to have many more than 1 local maximum. Since examination of the data reveals that open interest has only l local maximum on any one day, it is reasonable to require $\because(\tau)$ to have this property also. Essentially, what is required is some sort of smoothing operation to remove the small irregularities in $W(T)$; a very simple and effective method to accomplish this smoothing is a moving average. In this case, an eleven-period centered moving average sufficed to give $W(T)$ the required shape: First rising to a maximum, then monotonically falling, eventually to zero. Figure 1 displays (iT) yraphically.

Note that "Sample No. 2" starts when there was still a price ceiling imposed on the May 1951 and July 1951 contracts. Ideally, the sample period should be moved to a later starting point, but the bias involved in starting on March 24 is undoubtedly smal1. Price changes are constrained to 0 only for the May contract, with the July contract displaying some variability. The price changes used in the analysis are weighted average, which always includes unconstrained contracts; less than $30 \%$ of the volume is traded in May and July 1951 futures. Thus about $1 \%$ of the sample is affected by the price controls. The similarity of results for both samples indicates that the bias toward too many small price changes is negligible.

Also, weekends might be a source of error; intermittently throughout both sample periods the markets were open on Saturdays. The spectra for trading volume and price change indicate that the 6 -day cycle that might be introduced by Saturday trading is nonexistent. Similarly, no 5-day cycle is formed hy Lreating weekends the same as overnight periods. 


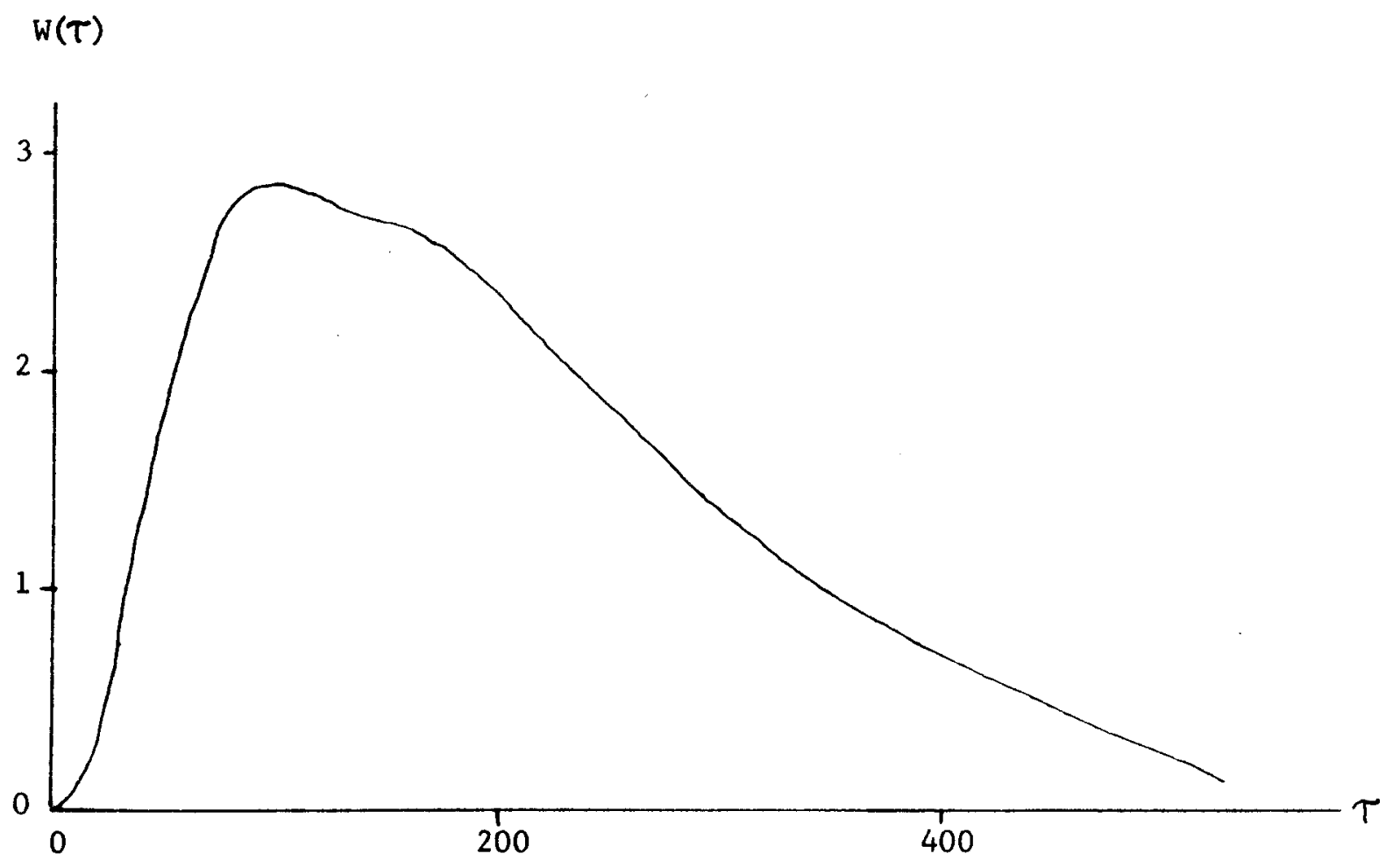

Figure Al

$W(T)$ as a function of $T$ 


\section{BIBLIOGRAPHY}

[1] Aitchison, J., and J. A. C. Brown, The Lognormal Distribution, Cambridge University Press, Cambridge, 1957.

[2] Anscombe, F. J., "Large Sample Theory of Sequential Estimation", Proceedings of the Cambridge Philosophical Society, Vol. 48 (1952), p. 600-607.

[3] Bachelier, L., Theory of Speculation, Gauthier-Villars, Paris, 1900.

[4] Billingsley, P., "Limit Theorems for Randomly Selected Partial Suns", Annals of Mathematical Statistics, Vol. 33 (1963), p. 85-92.

[5] Bochner, S., Harmonic Analysis and the Theory of Probability, University of California Press, Berkeley, 1960.

[6] Clark, P. K. A Subordinated Stochastic Process Model of Cotton Futures Prices, unpublished Ph. D. dissertation, Harvard University, May, 1970.

[7] Cooley, J. W., and J. W. Tukey, "An Algorithm for the Machine Calculation of Complex Fourier Series", Mathematics of Computation, Vol. 19 (1965), p. 297-301.

[8] Cootner, P. H., ed., The Random Character of Stock Market Prices, M.I.T. Press, Cambridge, Mass., 1964.

[9] Feller, W. , An Introduction to Probability Theory and Its Application, Vol. II, John Wiley and Sons, New York, 1966.

[10] Gnednenko, B. V., and A. N. Kolmogorov, Limit Distributions for Sums of Independent Random Variables, Addison-Wesley, Cambridge, Mass., 1954.

[11] Kenda11, M. G., "The Analysis of Economic Time Series: Part I, Prices", Journal of the Royal Statistical Society, Vol. 96 (1953), p. 11-25.

[12] Levy, P., Calcul des Probabilités, Gauthier-Villars, Paris, 1925.

[13] Loève, M. M. Probability Theory, Van Nostrand, New York, 1955.

[14] Mandelbrot, B., "The Variation of Certain Speculative Prices", Journal of Business, Vol. 36 (1963), p. 394-419.

[15] Mandelbrot, B. and H. Taylor, "On the Distribution of Stock Price Differences", Operations Research, Vol. 15 (1967), p. 1057-1062. 
[16] Renyi, A., "On the Asymptotic Distribution of a Sum of a Random Number of Independent Random Variables", Acta Mathematica, (Hungary), Vol . 8 (1957), p. 193-199.

[17] Renyi, A., "On the Central Limit Theorem for a Random Number of Random Variables", Acta Mathematica, (Hungary), Vol. 11 (1960), p. 97-102.

[18] Robbins, H., "The Asymptotic Distribution of the Sum of a Random Number of Randon Va iables", Bulletin of the American Mathe-. matical Society, Vol. 54 (1948), p. 1151-1161.

[19] United States Commodity Exchange Administration, Trade in Cotton Futures, Washington, D. C. 